\title{
Identification of differences in immunotherapy knowledge and practice patterns among oncologists from six European countries
}

\author{
IVAN MARQUES BORRELLO ${ }^{1}$, MADELINE MAG SCHAFFER ${ }^{2}$, \\ ELENA ROEHRL $^{2}$ and JESSICA FALCO MARSHALL ${ }^{3}$ \\ ${ }^{1}$ Sidney Kimmel Comprehensive Cancer Center, Johns Hopkins University, Baltimore, MD 21231, USA; \\ ${ }^{2}$ European Institute for Medical and Scientific Education, 1010 Vienna, Austria; \\ ${ }^{3}$ Advanced Studies in Medicine, Somerville, NJ 08876, USA
}

Received August 07, 2013; Accepted January 08, 2014

DOI: $10.3892 / \mathrm{mco} .2014 .242$

\begin{abstract}
The treatment of cancer has changed significantly over the past decade, from a treatment paradigm based primarily on surgery, chemotherapy and radiotherapy, to the development of targeted therapies involving tumor-specific signaling pathways, as well as the immune system. Recent developments have demonstrated the significant clinical benefits of immunotherapy that are quickly being established as standard treatments for certain malignancies. In this study, we demonstrated that clinical practice is not keeping pace with advancing immuno-oncology principles and research. We further demonstrated that the incorporation of immunotherapeutic approaches into the treatment of cancer patients varies widely, not only between developed and developing countries, but also between countries in specific geographical areas that have experienced similar cultural and economic development. We developed a survey to assess the deficiencies in scientific understanding, access to information and treatment options and investigated the differences in clinical practice behaviors among oncologists from six European countries: France, Germany, Greece, Italy, Spain and the United Kingdom. Our data demonstrated significant differences in all the categories assessed. Therefore, we concluded that there is a need to develop similar assessment tools to identify care imbalances, so that initiatives may be developed to correct care inequities that ultimately affect patient outcomes worldwide.
\end{abstract}

\section{Introduction}

The development of novel immune-based therapies for cancer has grown exponentially since the introduction of interleukin-2 in 1985 (1) and rituximab, the first monoclonal antibody

Correspondence to: Dr Jessica Falco Marshall, Advanced Studies in Medicine, 166 West Main Street, PO Box 340, Somerville, NJ 08876, USA

E-mail: jmarshall@asimcme.com

Key words: cancer immunotherapy, monoclonal antibodies, cancer vaccines
(mAb) used in the treatment of cancer patients, in $1997(2,3)$. Although our understanding of tumor immunology is evolving, the currently accepted paradigm is that immuno-oncology and immunotherapy comprise a clinical subspecialty involving strategies that target the immune system, either actively or passively, to generate or augment antitumor immunity $(4,5)$. As such, immunotherapy differs from chemotherapy, which targets the tumor cell itself by affecting cell growth or survival. Agents specifically recognized as immunotherapies include mAbs, growth factors and vaccines that boost or restore the immune system to produce antitumor responses (6-8).

Recent advances in immunotherapeutic approaches, such as cancer vaccines and checkpoint inhibitors, add to the complexity of integrating and/or sequencing these therapies. The first therapeutic cancer vaccine (sipuleucel-T) for the treatment of asymptomatic or minimally symptomatic metastatic, castration-resistant (hormone-refractory) prostate cancer was approved by food and drug administration (FDA) in 2010 (9). In 2011, FDA and European Union (EU) marketing approvals were granted to ipilimumab for the treatment of patients with metastatic melanoma $(10,11)$. Several other cancer immunotherapies, including therapeutic vaccines, are currently at various stages of development and investigation. All these factors contribute to the significant increase in the publications regarding immune-based treatments over the past several years. The challenge lies with ensuring this information is being conveyed to practicing oncologists in a manner that permits them to ultimately integrate these approaches into treatment regimens for their patients.

The inequity of cancer care among developed and developing countries is well-known (12); however, due to various reasons, healthcare systems may also vary significantly among developed countries. Amongst the first steps in correcting the inequities of cancer care should be identifying the deficiencies in scientific understanding, improving access to information and treatment options and eliminating the differences in clinical practice behaviors worldwide. Assessing the true impact of these differences on practicing oncologists is a prerequisite to developing customized strategies to help clinicians worldwide access the most current and effective treatment practices for their patients. Thus, it was crucial to develop and test an assessment tool with the potential to yield significant insight into clinical oncology practice standards of a specific geographical area. 


\section{Materials and methods}

Developers. This survey was developed through a collaboration between the Johns Hopkins University School of Medicine, Advanced Studies in Medicine and the European Institute for Medical and Scientific Education (EIMSED). Due to the current evolution in cancer therapy paradigms, we decided to focus on emerging treatment strategies in immuno-oncology and cancer immunotherapies. The Johns Hopkins University School of Medicine in the United States identified and emphasized specific clinical topics that are important to assess within this field. EIMSED selected Europe as the test area, targeting six representative countries: France, Germany, Greece, Italy, Spain and the United Kingdom (UK). EIMSED assisted with the development of data capture methods and survey execution.

Topics. The following topics relevant to immuno-oncology were selected for inclusion in the survey: basic scientific knowledge of immunotherapy, carcinogenesis as it relates to the immune system, mechanisms of chemoresistance, immunotherapy as adjuvant therapy, mAbs in cancer immunotherapy, T-cell approach in cancer immunotherapy, vaccines in cancer immunotherapy, vaccines to prevent infection by carcinogenic viruses, cytokines in cancer treatment, cytotoxic T-lymphocyte antigen 4 (CTLA-4), programmed cell death protein 1 (PD-1), immuno-biomarkers as predictors of response, tumor flare reactions (TFRs), side effects of cancer immunotherapy, Response Evaluation Criteria in Solid Tumors (RECIST), immune-related RECIST (iRECIST).

Sample. A sample size of $\sim 30$ oncologists per country was predetermined as being sufficient to yield reliable results. The physicians were randomly selected from urban centers, smaller cities and rural sites where possible (Table I). We employed a triangulated design to facilitate the validation of data and avoid complications in homogeneity. To assess the individual physician needs, as well as the needs of the profession, we engaged in a pre-survey market analysis in each country to establish the health of the general population, healthcare delivery characteristics and the path and status of general and continuing medical education (CME).

Self-evaluation. For the survey, the oncologists were asked to evaluate their personal knowledge and information level; relevance and knowledge in daily practice; and implementation of knowledge, skills and behavior in daily practice for each of the topics listed above. The participants were asked to utilize a rating scale of 1 to 5 , within 5 reflecting the status of being the most informed. The same rating scale was used to reflect the significance or relevance of each topic with their daily practice and reveal the extent to which knowledge of each topic could be implemented into their clinical practice setting. In an open discussion, the oncologists were queried to reveal more in-depth details of their opinions and treatment behaviors on immunological topics as follows: i) in which situations would oncologists consider the use of immunotherapy as adjuvant therapy; ii) their knowledge regarding the mechanisms of action of the mAbs used in immunotherapy, including naming specific mAbs they were familiar with; iii) their knowledge of the targets/receptors of the mAbs trastuzumab, ipilimumab,
Table I. Demographic characteristics of the survey sample.

Characteristics

Percentage $^{\mathrm{a}}(\mathrm{n}=169)$

$\begin{array}{lr}\text { Location } & \\ \text { Rural } & 1 \\ \text { Smaller city } & 33 \\ \text { Larger city } & 66 \\ \text { Duration of practice, years } & \\ 1-10 & 41 \\ 11-20 & 37 \\ >20 & 22\end{array}$

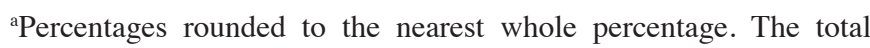
number of surveyed European oncologists was 169.

rituximab and bevacizumab and their indications; iv) identification of specific immune tolerance checkpoint inhibitors directed against the immune system; v) their knowledge level regarding sipuleucel-T and its indication; vi) their knowledge level regarding vaccine approaches that are currently under investigation; vii) their knowledge of the differences between preventive and therapeutic vaccines; viii) their level of knowledge regarding evaluating immunotherapy-induced lesions and TFR and their experience with TFRs in practice; ix) differences between RECIST and iRECIST criteria and their knowledge of why iRECIST criteria is relevant for current oncological practice; and $\mathrm{x}$ ) barriers that may prevent them from using approved cancer immunotherapies.

Demographics. The physicians were asked a series of demographic questions regarding their background and practice characteristics: their practice setting, specialization and number of years they have been practicing. They were also queried on their preferences regarding CME. Additional open questions on relevant topics during focus groups enabled the participants to disclose their professional challenges, barriers, awareness and practices on topics such as mAbs, cancer vaccines and iRECIST.

Data collection and analysis. Quantitative and qualitative data were collected utilizing two complementary methods. Between August, 2012 and October, 2012, we conducted computer-assisted telephone interviews with $\sim 30$ physicians in each targeted country, utilizing a formatted questionnaire. Additionally, in each country, a face-to-face focus group, including 4-6 representative oncologists, allowed for analysis, interpretation and open discussion of the topics included in the questionnaire. In conducting the survey, EIMSED cooperated with local scientific partner institutes in all six countries to ensure accurate translation of the questionnaire into the native language and to assist with the recruitment of the sample groups, interviews, data collection and translation of the assessed data. Telephone interviews and focus groups were conducted in the country's native language. This was a descriptive study using arithmetic averages and median. The NIPO-Diana software (NIPO software, Amsterdam, The Netherlands) was utilized in data recording and analysis. 
Table II. Oncologists self-reported as well-informed in immuno-oncology and cancer immunotherapies.

\begin{tabular}{|c|c|c|c|c|c|c|}
\hline \multirow[b]{2}{*}{ Topics } & \multicolumn{6}{|c|}{ Percentage of oncologists self-assessed as well-informed ${ }^{a}$} \\
\hline & $\begin{array}{l}\text { France } \\
(n=30)\end{array}$ & $\begin{array}{c}\text { Germany } \\
\left(\mathrm{n}=19^{\mathrm{b}}\right)\end{array}$ & $\begin{array}{l}\text { Greece } \\
(n=30)\end{array}$ & $\begin{array}{c}\text { Italy } \\
(\mathrm{n}=30)\end{array}$ & $\begin{array}{c}\text { Spain } \\
(\mathrm{n}=30)\end{array}$ & $\begin{array}{c}\text { UK } \\
(n=30)\end{array}$ \\
\hline Monoclonal antibodies & 83 & 89 & 76 & 57 & 43 & 60 \\
\hline Carcinogenesis as it relates to the immune system & 37 & 10 & 44 & 40 & 20 & 43 \\
\hline Immunobiomarkers/predictors of response & 74 & 63 & 40 & 43 & 26 & 33 \\
\hline Mechanisms of chemoresistance & 53 & 31 & 53 & 56 & 50 & 50 \\
\hline Immunotherapy as adjuvant therapy & 27 & 52 & 66 & 60 & 43 & 26 \\
\hline Side effects of cancer immunotherapy & 40 & 53 & 50 & 50 & 43 & 37 \\
\hline RECIST & 90 & 58 & 87 & 80 & 83 & 80 \\
\hline iRECIST & 30 & 5 & 40 & 47 & 30 & 33 \\
\hline TFRs & 27 & 26 & 33 & 37 & 10 & 13 \\
\hline Vaccines as cancer immunotherapy & 16 & 21 & 43 & 34 & 13 & 13 \\
\hline Preventive vaccines & 53 & 32 & 74 & 50 & 33 & 16 \\
\hline T-cell approach/cancer immunotherapy & 37 & 42 & 50 & 33 & 16 & 27 \\
\hline Cytokines & 33 & 32 & 63 & 33 & 24 & 40 \\
\hline PD-1 antibodies & 3 & 10 & 27 & 34 & 0 & 20 \\
\hline CTLA-4 antibodies & 17 & 16 & 50 & 37 & 30 & 33 \\
\hline
\end{tabular}

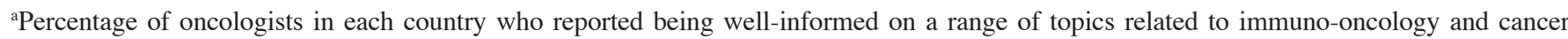
immunotherapies. Response ranges of 4 and 5 were considered to be well-informed and very well-informed. ${ }^{\text {bIt }}$ was difficult to accrue oncologists, as the survey period overlapped with the holiday season in Germany. Since those surveyed yielded similar answers and discussion, the results were tabulated and assessed utilizing the data from the 19 participants. RECIST, Response Evaluation Criteria in Solid Tumors; iRECIST, immune-related RECIST; TFRs, tumor flare reactions; PD-1, programmed cell death protein 1; CTLA-4, cytotoxic T-lymphocyte antigen 4.

\section{Results}

Similarities among countries. The results of the study were analyzed in accordance with the requirements of data protective directives. Certain similarities were observed among the European countries surveyed. For example, medical studies generally range between 5 and 6 years and medical oncology is usually included as part of the internal medicine training program. Medical oncology was officially recognized as a specialty by the EU as late as 2011 (13). Certain policy issues are common to all the EU countries, including the use of a centralized procedure for market authorization of new drugs. Another affecting factor is the detrimental effect of the economic downturn in the EU at the time this survey was conducted. Oncologists from all the countries reported the effect of the economic recession on healthcare, although to varying degrees. We observed certain country-specific results in terms of clinical knowledge; for example, there were variations in the self-assessment of oncologists as to how well-informed they are overall, regarding immuno-oncology and cancer immunotherapy (Fig. 1). Notably, oncologists in Spain consider themselves to have very little information on the topic as a whole.

Self-assessment. Only 35\% of the interviewed European oncologists considered themselves to be well-informed on immuno-oncology and cancer immunotherapy. The majority were more familiar and comfortable with the concepts of chemotherapy, radiotherapy and endocrine therapy. Moreover, clinically active oncologists in Europe tended to focus more on

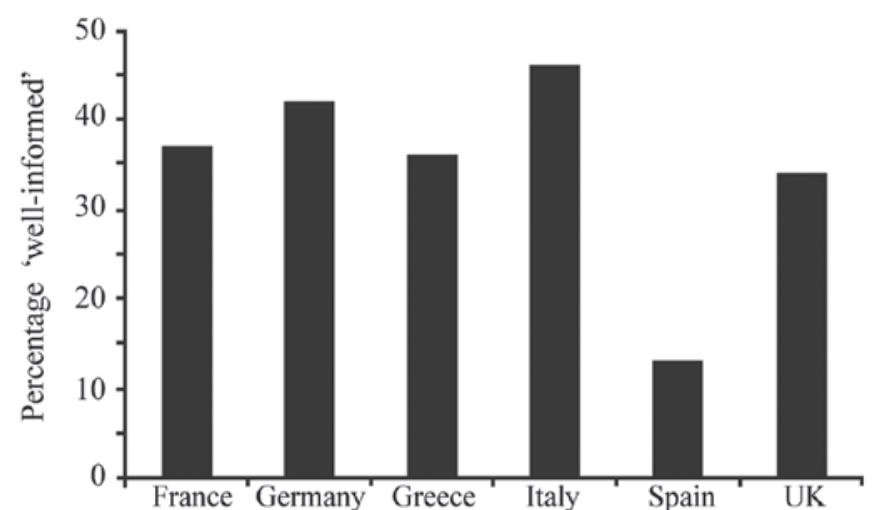

Figure 1. Oncologists' self-reported knowledge of immuno-oncology and cancer immunotherapies. UK, united kingdom.

the clinically relevant data and concepts rather than the biological rationale or mechanism(s) of action of these agents. The clinicians tended to associate immuno-oncology more with science and basic research rather than with clinical practice.

Topics of relevance. The oncologists from all six countries found the following topics most relevant: $\mathrm{mAbs}$ in cancer immunotherapy, RECIST and mechanisms of chemoresistance. The majority also found the following topics of moderate relevance: side effects of cancer immunotherapy (only $45 \%$ felt they were well-informed on this topic) and immuno-biomarkers as predictors of response (only $46 \%$ felt 
Table III. Barriers to the implementation of immunotherapies.

\begin{tabular}{|c|c|c|c|c|c|c|}
\hline Barriers & France & Germany & Greece & Italy & Spain & UK \\
\hline High therapy costs & 33 & 16 & 60 & 20 & 73 & 73 \\
\hline Lack of data/studies & 17 & 42 & 10 & 10 & 43 & 37 \\
\hline Lack of information on specific therapies & 13 & 5 & 13 & 3 & 20 & 17 \\
\hline Lack of access ${ }^{\mathrm{a}}$ & 10 & 47 & 33 & 10 & 47 & 7 \\
\hline No barriers & 37 & 37 & 20 & 40 & 7 & 13 \\
\hline
\end{tabular}

Values are presented as the percentages. ${ }^{2}$ Lack of access includes lack of marketing approval and lack of reimbursement. UK, United Kingdom.

Table IV. Oncologists' learning preferences.

\begin{tabular}{|c|c|c|c|c|c|c|}
\hline A, CME preferences & $\begin{array}{l}\text { France } \\
(\mathrm{n}=30)\end{array}$ & $\begin{array}{l}\text { Germany } \\
(\mathrm{n}=19)\end{array}$ & $\begin{array}{l}\text { Greece } \\
(n=30)\end{array}$ & $\begin{array}{l}\text { Italy } \\
(\mathrm{n}=30)\end{array}$ & $\begin{array}{l}\text { Spain } \\
(\mathrm{n}=30)\end{array}$ & $\begin{array}{c}\mathrm{UK} \\
(\mathrm{n}=30)\end{array}$ \\
\hline Live educational programming & 97 & 58 & 87 & 90 & 83 & 63 \\
\hline Online educational activities & 27 & 11 & 60 & 90 & 40 & 3 \\
\hline Smaller workshops & 70 & 68 & 77 & 80 & 87 & 43 \\
\hline Symposia & 20 & 32 & 27 & 23 & 13 & 30 \\
\hline Congresses (>100 participants) & 63 & 37 & 23 & 40 & 13 & 47 \\
\hline Local/European instructors & 80 & 64 & 90 & 23 & 94 & 54 \\
\hline $\begin{array}{l}\text { Competency and good teaching skills } \\
\text { as the only instructor requirements }\end{array}$ & 23 & 63 & 17 & 67 & 30 & 37 \\
\hline $\mathrm{B}$, Program timing and duration & $\begin{array}{l}\text { France } \\
(n=30)\end{array}$ & $\begin{array}{l}\text { Germany } \\
(\mathrm{n}=19)\end{array}$ & $\begin{array}{l}\text { Greece } \\
(n=30)\end{array}$ & $\begin{array}{l}\text { Italy } \\
(\mathrm{n}=30)\end{array}$ & $\begin{array}{l}\text { Spain } \\
(\mathrm{n}=30)\end{array}$ & $\begin{array}{c}\mathrm{UK} \\
(\mathrm{n}=30)\end{array}$ \\
\hline Weekdays, evenings & 20 & 53 & 43 & 0 & 60 & 50 \\
\hline Weekdays, daytime & 37 & 37 & 7 & 43 & 60 & 33 \\
\hline Weekends & 60 & 58 & 67 & 57 & 13 & 17 \\
\hline One to a few hours in length & 30 & 68 & 17 & 7 & 30 & 20 \\
\hline Full-day programs & 43 & 5 & 33 & 37 & 63 & 57 \\
\hline 2-3 days & 23 & 16 & 47 & 47 & 10 & 13 \\
\hline
\end{tabular}

The values are presented as percentages. The total sum of the answers by category may not equal $100 \%$, as the oncologists were permitted to give more than one answer or to not have a preference. CME, continuing medical education. UK, United Kingdom

they were well-informed on this topic). A summary of the oncologists' self-assessment regarding their knowledge on the various topics within the survey is presented in Table II.

The surveyed oncologists placed low relevance on and were considerably less informed about TFRs, cancer vaccines and the T-cell approach to cancer immunotherapy. In Europe, $63 \%$ of the oncologists reported never having received information on how TFRs should be evaluated, although $43 \%$ said they had been confronted with TFRs in their practice. With the exception of France, less than half of the oncologists were able to cite types of cancer for which TFRs were covered in the literature. Oncologists in France, Spain and the UK reported having the least amount of information on evaluating TFRs. Furthermore, $<15 \%$ of the oncologists in Spain and the UK reported never encountering a TFR in practice. However, despite having little information on how to evaluate TFRs and immunotherapy-induced lesions in
France, $53 \%$ of the oncologists reported having been confronted with TFRs in their practices and $70 \%$ reported having read specific information in the literature.

Only $33 \%$ of the surveyed oncologists considered themselves to be well-informed on vaccines for cancer immunotherapy. Furthermore, $<14 \%$ of the oncologists from the six countries surveyed knew about tumor-cell based vaccines, viral vector vaccines, dendritic cell vaccines and peptide or protein vaccines. Although at the time of the survey sipuleucel-T (an autologous dendritic cell vaccine approach) had not yet been approved for marketing in the EU, only $21 \%$ of the oncologists were able to identify it as a novel therapy for castration-resistant prostate carcinoma. Conversely, although interferons and interleukins have been on the market for a long time, only $46 \%$ of the surveyed oncologists reported being well-informed on cytokines and only $22 \%$ reported being well-informed on anti-PD-1. 
There is greater knowledge on vaccines that prevent infections by carcinogenic viruses; $92 \%$ of the oncologists knew about the vaccine against human papillomavirus, a cause of cervical cancer. However, only $36 \%$ were aware of hepatitis B vaccination for the prevention of liver cancer and $56 \%$ of the surveyed oncologists said they would like to know more about preventive vaccines.

On the general topic of mAbs, $67 \%$ of the oncologists felt well-informed, although the extent of knowledge varied from antibody to antibody, with greater recognition of targeted mAbs, which have been available for a longer period of time (e.g., trastuzumab, rituximab and bevacizumab) compared to ipilimumab, which received marketing authorization in the EU in May, 2011 (11). Overall, the therapeutic mechanism of the action of ipilimumab (anti-CTLA-4) was unknown to $47 \%$ of the surveyed oncologists and $31 \%$ were not familiar with this agent's indication for unresectable or metastatic melanoma. Oncologists in Spain and the UK appeared to be particularly more informed on this topic; 70\% of the Spanish and 77\% of the UK oncologists could identify CTLA-4 as the receptor/target of ipilimumab and 93 and 83\%, respectively, knew its indication.

Barriers. Oncologists in Europe cited several barriers to the implementation of immuno-oncological approaches in practice. Generally, the high cost of such therapies, particularly when their benefits have not been clearly determined, is a significant hindrance (Table III). This situation is pervasive throughout European countries and frequently results in barriers to access and reimbursement. Oncologists from all six countries cited a lack of regulatory and/or health system approvals as a barrier to implementation of immuno-oncology practices. They also cited a personal lack of knowledge and confidence in the therapies, in toxicity management and in the appropriateness of the objective (cost vs. age). Greek oncologists in particular cited their country's economic condition as a barrier to the implementation of such therapies.

Learning preferences. We also surveyed oncologists in each country on their preferences for medical education covering these topics. Overall, the oncologists were eager for information on immuno-oncology; however, they were unanimous in stressing the point that each CME encounter must aim at communicating content that is relevant for their practice and is indication-specific. However, preferences among countries varied (Table IV).

\section{Discussion}

Immuno-oncology topics were selected for this survey to gain a broad overview from oncologists regarding this clinical subject area, as well as to assess their access to and acceptance of novel therapeutic strategies. The results of the survey demonstrated significant gaps in knowledge and understanding of this relatively new and rapidly expanding field and this survey clearly established that gaps in knowledge and behaviors vary widely across European countries. In fact, there is broad divergence from the currently accepted definition of cancer immunotherapy in the UK, where oncologists do not associate $\mathrm{mAbs}$ with immunotherapy; they refer to them as newer forms of chemotherapy and perceive immuno-oncology as an integral component of cancer therapy as a whole. Conversely, oncologists in Italy and Greece, despite considering themselves well-informed on the topic, felt immunotherapy was not yet relevant to clinical practice.

Certain other responses demonstrate a lack of knowledge of the principles of immunotherapy. Clinicians are erroneously applying chemotherapy methodologies to immunotherapy when an entirely new paradigm is required (6). There are characteristics unique to cancer immunotherapy and European oncologists do not appear to have a substantial understanding of these differences. An example is in the evaluation of TFRs. In the case of conventional cytotoxic chemotherapeutic agents, treatment response is evident early during the course of therapy when these agents are effective; thus, an observable increase in tumor growth or new lesions signals progressive disease. As immunotherapy acts by enhancing the host's antitumor immune responses, there is often an initial increase in the tumor burden after the initiation of immunotherapy. A period of tumor flare may be followed by the development of durable stable disease or disease responsiveness. This characteristic of immunotherapy is not normally observed with traditional chemotherapy. This has led to the development of novel methods of measuring disease response in patients undergoing treatment with immune-based therapies (14). Despite this new response system, $63 \%$ of the European oncologists have no information on how they should evaluate TFRs.

Another point supporting the lack of understanding of the differences between chemotherapy and immunotherapy arose during discussions on RECIST and iRECIST criteria. RECIST is a set of established criteria or standards, internationally recognized for evaluating patient response, stability and progression in clinical trials and in the clinical practice. Originally published in 2000 as a joint effort of the European Organization for Research and Treatment of Cancer, the National Cancer Institute of the United States and the National Cancer Institute of Canada Clinical Trials Group, RECIST has traditionally been utilized in the evaluation of response to chemotherapy. However, tumor response to immunotherapy can be quite different; therefore, a new set of criteria for evaluating response and for clinical data endpoints was required. The iRECIST update was published in 2009 and included criteria necessary for evaluating immunotherapy activity in solid tumors $(14,15)$. However, less than half of the surveyed European oncologists reported an understanding of iRECIST and their relevance to cancer immunotherapy.

Certain factors emerged in conducting this survey, which have the potential to introduce bias. Although we attempted to enroll oncologists from varying geographic locations and practice types, only $1 \%$ of the surveyed oncologists were practicing in rural areas. However, due to of the smaller geographic size of European countries, most medical specialists are located in smaller cities and larger urban areas that are fairly easy to access for the majority of patients, including those living in more rural settings. Additionally, the target number of participants from each country was determined to be 30 oncologists. However, only 19 oncologists from Germany participated in the survey. For a variety of reasons, it was difficult to accrue a full complement of German oncologists. In the interest of completing this assessment in a timely fashion, we reviewed responses and discussions and identified patterns that may be 
reflective of responses that would occur in a full complement of 30 oncologists. Therefore, a decision was made to tabulate and assess the data for Germany based on those 19 participants.

In the process of conducting the survey, it became clear that there was no standard definition for immune biomarkers, immuno-oncology and immunotherapy in the minds of the European oncologists. For the purposes of this report, immuno-biomarkers were defined as markers that predict response to a therapy falling under a broad definition of immunotherapy, including mAbs and vaccines. Although some may argue that the anticancer activity of some mAbs is unrelated to directly enhancing or repressing an immune response, we made no attempt to make such distinctions or define the terms for the survey participants. The intent was to phrase survey questions in a manner so as not to bias the participants in any way and to stimulate free and open discussion in the focus groups. Inherent discrepancies in the definition of terms has the potential to slightly skew the data. In our survey, for example, French and Spanish oncologists revealed that they do not consider mAbs to be immunotherapy. Furthermore, although European oncologists considered themselves to be well-informed on mAbs, their responses as a whole revealed a general lack of knowledge regarding therapeutic vaccines. Consequently, we believe that, rather than confounding the assessment, minimizing bias in this situation may have led to a clearer insight into the deficits of clinicians' understanding of immuno-oncology and immunotherapies.

Oncologists in Spain displayed a significantly lower level of understanding of immuno-oncology and cancer immunotherapies compared to oncologists from the other surveyed countries. This was supported through survey responses across the board and in the focus groups. We are left to ponder the potential factors that may be contributing to this condition. Half of those surveyed had been practicing for $\leq 10$ years; therefore, they were likely exposed to some immuno-oncology information during medical training. Further investigation is required to identify contributing factors and their potential impact.

Furthermore, the number of years oncologists had been practicing had the potential to affect the physician responses. Those practicing for only a short time likely had medical training more recently and, therefore, the opportunity to acquire more knowledge on targeted therapies and immunotherapy, as well as the understanding of the clinical differences between the two classes. However, this appears to have had little effect on the results. Approximately two-thirds of the surveyed German oncologists had been practicing for $\leq 10$ years and, although they had significant knowledge of mAbs, they lacked understanding of newer immunotherapy approaches.

This survey supports several observations. The rapid transformation currently occurring in the field of oncology may be further complicating worldwide inequities in cancer care. There is significant diversity regarding the knowledge, understanding, viewpoints and behaviors among oncologists on topics related to immuno-oncology and cancer immunotherapies. For clear reasons, cancer care inequities are expected to exist between developing and developed countries; however, these inequities are occurring even in geographic areas with similar economic and scientific development, policy making, education and general population health. We concluded that there is a need for assessment tools to identify the elements of care imbalances and to develop initiatives aimed at correcting these imbalances. Ultimately, these initiatives must be judiciously customized to fit the specific needs of the different geographic areas and specific countries targeted.

\section{Acknowledgements}

We would like to acknowledge the support of a Bristol-Meyers Squibb Independent Medical Education Grant awarded under the direction of the late Christian Reboulleau. We also acknowledge Joanna Shields for assisting with the manuscript development, Robin Soboti for contributing to the survey content development, Reshma Carter, PharmD, for valuable discussions on the manuscript development, Todd Dorman, FCCM, from the Johns Hopkins University School of Medicine Office of CME, Cathy Zehr for assisting with figure formatting and Lisa Mangione for editorial assistance.

\section{References}

1. DeVita VT Jr and Rosenberg SA: Two hundred years of cancer research. N Engl J Med 366: 2207-2214, 2012.

2. US Department of Health and Human Services: First monoclonal antibody approved to treat cancer [press release], November 26, 1997: http://archive.hhs.gov/news/press/1997pres/971126. html accessed June 17, 2013

3. Boyiadzis M and Foon KA: Approved monoclonal antibodies for cancer therapy. Expert Opin Biol Ther 8: 1151-1158, 2008.

4. Mellman I, Coukos G and Dranoff G: Cancer immunotherapy comes of age. Nature 480: 480-489, 2011.

5. Finn OJ: Immuno-oncology: understanding the function and dysfunction of the immune system in cancer. Ann Oncol 23: viii6-viii9, 2012.

6. Hoos A and Britten CM: The immuno-oncology framework: enabling a new era of cancer therapy. Oncoimmunology 1 : 334-339, 2012.

7. Borghaei H, Smith MR and Campbell KS: Immunotherapy of cancer. Eur J Pharmacol 625: 41-54, 2009.

8. Butterfield LH, Disis ML, Khleif SN, Balwit JM and Marincola FM: Immuno-oncology biomarkers 2010 and beyond: perspectives from the iSBTc/SITC biomarker task force. J Transl Med 8: 130, 2010.

9. FDA approval letter to Dendreon Corporation, April 29, 2010: http://www.fda.gov/BiologicsBloodVaccines/ CellulargeneTherapyProducts/ApprovedProducts/ucm210215.htm accessed June 17, 2013

10. US Department of Health and Human Services, Food and Drug Administration: BLA approval letter, YERVOY (ipilimumab), March 25, 2011: http://www.accessdata.fda.gov/drugsatfda_docs/ appletter/2011/125377s000ltr.pdf accessed June 17, 2013

11. Committee for Medicinal Products for Human Use, European Medicines Agency: Assessment Report for Yervoy (ipilimumab). Procedure number: EMEA/H/C/002213, May 19, 2011: http:// www.ema.europa.eu/docs/en_GB/document_library/EPAR_Public_assessment_report/human/002213/WC500109302.pd $\bar{f}$ accessed June 17, 2013

12. Abdel-Wahab M, Alfonso R, Anacak Y, et al: Human Health Reports No. 3: Inequity in cancer care: a global perspective. International Atomic Energy Agency, Vienna, Austria, pp1-51, 2011.

13. Casali PG: Medical oncology: the long-awaited prize of recognition. Ann Oncol 8: 1695-1697, 2011

14. Wolchok JD, Hoos A, O'Day S, et al: Guidelines for the evaluation of immune therapy activity in solid tumors: immune-related response criteria. Clin Cancer Res 15: 7412-7420, 2009.

15. Eisenhauer EA, Therasse P, Bogaerts J, et al: New response evaluation criteria in solid tumors: Revised RECIST guideline (version 1.1). EJC 45: 228-247, 2009. 